intervention is impossible. Chemotherapy has shown no beneficial outcome unless malignancy is present. The overall prognosis for IPT is excellent. Most recurrences will arise during the first year after initial therapy. Only a few reports showed late-onset recurrences.

\section{References}

1. Dunnick NR. Image interpretation session: 1999. Inflammatory myoblastic pseudotumor of the trachea (plasma cell granuloma). Radiographics. 2000;20:274-6.

2. Cerfolio RJ, Matthews TC. Resection of the entire left mainstem bronchus for an inflammatory pseudotumor. Ann Thorac Surg. 2005;79:2127-8.
3. Amir R, Danahey D, Ferrer K, Maffee M. Inflammatory myofibroblastic tumor presenting with tracheal obstruction in a pregnant woman. Am J Otolaryngol. 2002;23:362-7.

4. Browne M, Abramson LP, Chou PM, Acton R, Holinger LD, Reynolds M. Inflammatory myofibroblastic tumor (inflammatory pseudotumor) of the neck infiltrating the trachea. J Pediatr Surg. 2004;39:e1-4.

5. Bumber Z, Jurlina M, Manojlovic S, Jakic-Razumovic J. Inflammatory pseudotumor of the trachea. J Pediatr Surg. 2001;36:631-4.

6. Nikanne E, Sopanen J, Seppa A. Inflammatory pseudotumor of the trachea. Otolaryngol Head Neck Surg. 2004;130:274-6.

7. Coffin CM, Watterson J, Priest JR, Dehner LP. Extrapulmonary inflammatory myofibroblastic tumor (inflammatory pseudotumor). A clinicopathologic and immunohistochemical study of 84 cases. Am J Surg Pathol. 1995;19: 859-72.

\title{
Infective mitral valve myxoma with coronary artery embolization: Surgical intervention followed by prolonged survival
}

Feng Yao, MD, Zhi-yun Xu, MD, Yan-lin Liu, MS, and Lin Han, MD, Shanghai, People's Republic of China

Three-quarters of primary cardiac neoplasms are benign; nearly half of benign heart tumors are myxomas, which have an estimated incidence of 0.5 per million population per year. ${ }^{1,2}$ Roughly $75 \%$ of myxomas originate from the left atrium, and $15 \%$ to $20 \%$ originate from the right atrium. Myxomas of the heart valves are exceedingly rare. ${ }^{1}$ Myxomas may simulate infective endocarditis, but rarely are they actually infected.

We report a case of a 12-year-old child with an infective myxoma originating from the ventricular side of the anterior mitral leaflet with a coronary artery embolism.

\section{CLINICAL SUMMARY}

A 12-year-old girl from a rural area of mainland China with a 9-year history of recurrent intermittent fevers and night sweats was admitted to our hospital on April 4, 2001. During the 9 years prior to admission, the girl was diagnosed with an upper respiratory tract infection each time she developed high fever. This diagnosis was given because no other positive evidence could be found, and the girl was treated with empirical antibiotics. At the time of admission,

From the Department of Cardiothoracic Surgery, Changhai Hospital, Second Military Medical University, Shanghai, People's Republic of China.

Received for publication Nov 24, 2007; revisions received Jan 8, 2008; accepted for publication Jan 15, 2008.

Address for reprints: Zhi-yun Xu, MD, Department of Cardiothoracic Surgery, Changhai Hospital, 174 Changhai Road, Shanghai 200433, People's Republic of China (E-mail: zhiyunx@hotmail.com).

J Thorac Cardiovasc Surg 2009;137:749-51

$0022-5223 / \$ 36.00$

Copyright (C) 2009 by The American Association for Thoracic Surgery

doi:10.1016/j.jtcvs.2008.01.050 however, she had maintained fevers as high as $103.5^{\circ} \mathrm{F}$ $\left(39.5^{\circ} \mathrm{C}\right)$ for 3 weeks. These fevers were unresponsive to high doses of intravenous penicillin. Moreover, the patient had developed severe dyspnea and lower extremity edema. There was a III/6 soft, blowing, systolic murmur at the apex on auscultation.

Neisseria lactamica was isolated from blood cultures on 2 consecutive days, and an emergency echocardiogram was obtained, which revealed a mass in the left ventricle attached to the anterior mitral leaflet, with severe mitral regurgitation (Figure 1).

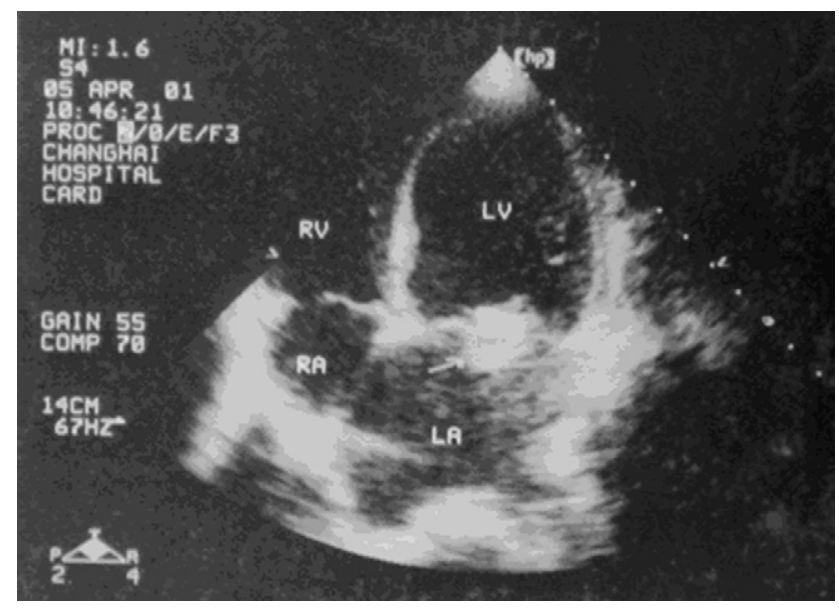

FIGURE 1. Transthoracic echocardiography in the 4-chamber apical view, revealing a left ventricular mass (arrow) attached to the anterior mitral leaflet. $L V$, left ventricle; $R V$, right ventricle; $L A$, left atrium; $R A$, right atrium. 


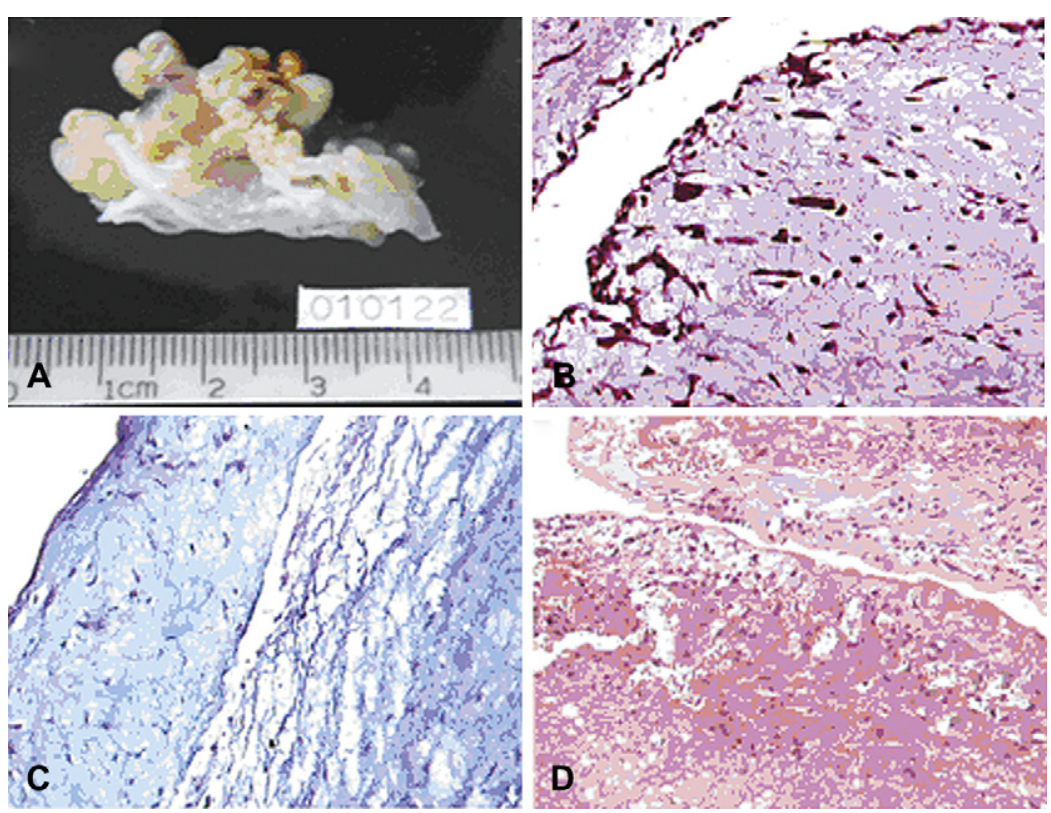

FIGURE 2. A, Pathologic specimen of the removed mitral valve covered by the myxoma and part of the papillary muscle. B, The majority part of the specimen exhibits typical characteristics of myxoma (ie, eristic acid-mucopolysaccharide matrix) and embedded polygonal cells (hematoxylin and eosin staining, magnification $\times 200$ ). C, Acid-mucopolysaccharide matrix (periodic acid Schiff staining, magnification $\times 200$ ). $D$, The minority part of the specimen shows acute inflammation and extensive necrosis (hematoxylin and eosin, magnification $\times 200$ ).

Cardiac surgery with cardiopulmonary bypass was performed 1 week later. A $3.5 \times 2.5-\mathrm{cm}$ grapelike tumor originating from the ventricular side of the anterior mitral leaflet and papillary muscles was resected (Figure 2), and the mitral valve was replaced with a 27-mm St Jude Medical mechanical prosthetic valve (St Jude Medical, Inc, St Paul, Minn). However, the patient could not be weaned from cardiopulmonary bypass due to poor cardiac contractility. A coronary artery examination showed that the left anterior descending (LAD) branch of the coronary artery had been completely occluded by a tumor fragment when it was incised. Emergency coronary artery bypass grafting surgery was performed using a saphenous vein as a grafting vessel and the LAD as the target vessel. After this procedure, the patient displayed satisfactory cardiac output and was weaned from cardiopulmonary bypass uneventfully.

Microscopic pathology was consistent with a typical myxoma but also demonstrated acute inflammation (Figure 2). However, Gram staining and tissue cultures were negative. Postoperatively, the patient received 4 weeks of intravenous ceftriaxone therapy. After discharge, an echocardiogram was performed annually. The latest follow-up echocardiogram, performed in August 2007, did not show recurrent myxoma.

\section{DISCUSSION}

Although myxomas are rare in the cardiac surgical population, they are the most common benign neoplasms of the heart and represent the vast majority of all primary cardiac tumors. ${ }^{3}$ Cardiac myxomas may simulate infective endocarditis, although they are rarely actually infected. ${ }^{4}$ The risk of true infection still exists, however, because a myxoma presents a perfect infection site. A three-level definition of infected cardiac myxomas has been proposed. ${ }^{5}$ Classifications include definite, probable, and possible infections and are based on clinical and pathologic evidence. Criteria for "definite" infected myxomas include documented myxoma by pathology accompanied by either positive blood cultures and inflammation or microorganisms seen on pathology. In our case, the documented myxoma as evidenced by clinical examination, pathology, and positive blood cultures qualify this case as a "definite" infected cardiac myxoma.

Systemic embolization occurs in $30 \%$ to $40 \%$ of patients with myxomas, although the incidence of coronary embolization in patients with myxomas is only $0.06 \% .^{5}$ Although special care was taken in our case to avoid embolization of cardiac myxoma fragments perioperatively, embolization did occur during surgery procedures before crossclamping. The following two factors may account for this. First, mitral valve myxomas present a major risk of embolization because of both the high mobility of their implantation areas and the high pressure inside the left ventricle. Second, infected cardiac myxomas are more dangerous than noninfected myxomas: their incidence of embolization is increased two- to threefold. ${ }^{5}$

We conclude that infective mitral valve myxoma is an extremely rare condition. Because it has such a high incidence of embolization, emergency surgery should be done to 
remove this type of myxoma once it is diagnosed. During surgical procedures, careful measures should be taken to prevent fragments of the tumor from breaking away.

\section{References}

1. Reynen K. Cardiac myxomas. New Engl J Med. 1996;333:1610-7.
2. MacGowan SW, Sidhu P, Aherne T, et al. Atrial myxoma: national incidence diagnosis and surgical management. Ir J Med Sci. 1993;162:223-6.

3. Roschkov S, Rebeyka D, Mah J, et al. The dangers of cardiac myxomas. Prog Cardiovasc Nurs. 2007;22:27-30.

4. Vogt PR, Jenni R, Turina MI. Infected left atrial myxoma with concomitant mitral valve endocarditis. Eur J Cardiothorac Surg. 1996;10:71-3.

5. Revankar SG, Clark RA. Infected cardiac myxoma: case report and literature review. Medicine. 1998;77:337-44.

\section{Familial fetal-type rhabdomyoma of the tricuspid valve in the neonate: Malignant course for a benign disease}

Francesca Viscardi, MD, ${ }^{\mathrm{a}}$ Gabriella Errico, MD, ${ }^{\mathrm{b}}$ Nicola Schiavo, MD, ${ }^{\mathrm{c}}$ Paolo Biban, MD, ${ }^{\mathrm{b}}$ Alessandro Mazzucco, MD, ${ }^{\mathrm{a}}$ and Giovanni Battista Luciani, MD, ${ }^{\text {a }}$ Verona, Italy

\section{Supplemental material is available online.}

We report 2 brothers with prenatal diagnosis of tricuspid valve masses, both requiring aggressive intensive care resuscitation shortly after birth and surgical excision of the tumor, which proved to be a fetal-type rhabdomyoma. The unique pathologic and clinical features of this rare disease are discussed.

\section{CLINICAL SUMMARY}

Patient 1. The first child had a critical neonatal period due to severe tricuspid valve stenosis, caused by 3 obstructive cardiac masses and severe pulmonary hypertension. Treatment included balloon atrial septostomy to maintain cardiac output and mechanical ventilation, with prostaglandin E1 (PGE1) infusion and inhaled nitric oxide (NO) for 2 months after birth. Discharge therapy with sildenafil and bosentan was continued until the time of elective surgical resection of tricuspid valve masses (Figure 1, A), at 11 months of age. Histology showed only dystrophic calcification. Excellent tricuspid valve function and no recurrence of cardiac

\footnotetext{
From the Divisions of Cardiac Surgery, ${ }^{\mathrm{a}}$ Pediatrics, ${ }^{\mathrm{b}}$ and Pathology, ${ }^{\mathrm{c}}$ University of Verona, Verona, Italy.

Received for publication Nov 17, 2007; accepted for publication March 23, 2008.

Address for reprints: Giovanni Battista Luciani, MD, Division of Cardiac Surgery,

University of Verona, O. C. M. Piazzale Stefani 1, Verona, 37126, Italy (E-mail:

gbluciani@yahoo.com).

J Thorac Cardiovasc Surg 2009;137:751-3

$0022-5223 / \$ 36.00$

Copyright (c) 2009 by The American Association for Thoracic Surgery

doi:10.1016/j.jtcvs.2008.03.066
}

masses were documented at follow-up echocardiogram 1 year after operation. The child has no clinical nor laboratory evidence of tuberous sclerosis.

Patient 2. The younger brother also had severe tricuspid valve obstruction (functional atresia), due to a huge tricuspid valve mass, and pulmonary hypertension at birth, with echocardiographic evidence of adequate right-to-left shunting at the atrial septal level. Mechanical ventilation, PGE1 infusion, and inhaled NO failed to stabilize clinical status, and emergency surgical resection was required at 21 days of life (Figure 1,B). Despite satisfactory repair of tricuspid valve, extracorporeal membrane oxygenation (ECMO) support was necessary for refractory hypoxia. Six days after ECMO weaning, the neonate died due to sepsis and recurrent respiratory failure. Histology of the lesion evidenced fibromyxoid stroma and isolated spindle cells with eosinophilic cytoplasm (Figure 2, $A$ ); immunohistochemical staining was positive for desmin (Figure 2, $B$ ). The final pathologic diagnosis was calcified fetal rhabdomyoma of the tricuspid valve and recurrent pulmonary embolism, with no evidence of tuberous sclerosis.

\section{DISCUSSION}

The clinical and pathologic features of the 2 sibling patients herein are very uncommon. Considering the frequency of rhabdomyoma as cause of fetal and neonatal primary cardiac tumor, ${ }^{1}$ findings of large, hyperechogenic intracardiac masses suggested rhabdomyoma as presumptive diagnosis in both patients. On the contrary, tricuspid valve location, absence of tuberous sclerosis, ${ }^{1,2}$ no tendency toward regression, severe intracardiac obstruction with repeated pulmonary embolization, and, most unusually, familial recurrence brought the latter diagnosis into question. Although 